\title{
Chronic supplementation of a mushroom blend on oxygen kinetics, peak power, and time to exhaustion
}

\author{
Katie R Hirsch*, Meredith G Mock, Erica J Roelofs, Eric T Trexler, Abbie E Smith-Ryan \\ From The Twelfth International Society of Sports Nutrition (ISSN) Conference and Expo \\ Austin, TX, USA. 11-13 June 2015
}

\begin{abstract}
Background
Cordyceps militaris has been used in pre-workout supplement blends intended to improve aerobic performance. Mushroom blends containing Cordyceps may serve as an ergogenic aid by improving oxygen kinetics and delaying fatigue, but there is limited data on the effects of this ingredient during exercise. The purpose of this study was to determine the effects of 3 weeks of supplementation with a mushroom blend on oxygen kinetics, aerobic power, time to exhaustion, and lactate levels during high-intensity exercise.
\end{abstract}

\section{Methods}

In a double-blind placebo controlled design, recreationally active subjects $(\mathrm{n}=10$; mean $\pm \mathrm{SD}$; age $=21.4 \pm 2.4 \mathrm{yrs}$; height $=175.8 \pm 78 \mathrm{~cm}$; weight $=75.0 \pm 10.6 \mathrm{~kg})$ were randomly assigned to a mushroom $(\mathrm{MR} ; \mathrm{n}=6)$ or placebo $(\mathrm{PL} ; \mathrm{n}=5)$ treatment group. All subjects completed a maximal graded exercise test, 6 min sub-maximal cycle test, and 3 min all-out cycle test, each separated by at least $24 \mathrm{hrs}$. Maximal oxygen consumption $\left(\mathrm{VO}_{2} \mathrm{max}\right)$, time to exhaustion (TTE), and ventilatory threshold (VT) were determined via respiratory gas analysis during the maximal graded exercise test performed on a cycle ergometer. Lactate and oxygen saturation $\left(\mathrm{SPO}_{2}\right)$ were measured at 0 , 2, 3 and 6 min during the 6 min sub-max cycle test at a workload of $60 \%$ between $\mathrm{VT}$ and $\mathrm{VO}_{2}$ max. Peak power output (PP) was recorded during the 3 min all-out cycle test with a resistance of $4.5 \%$ of body weight. Subjects were given capsules containing either 1.3 grams of mushroom blend or 1.3 grams of maltodextrin (PL) to be taken

* Correspondence: ktrose23@live.unc.edu

Applied Physiology Laboratory, Department of Exercise and Sport Science, University of North Carolina at Chapel Hill, Chapel Hill, NC 27599, USA
3 times per day (4 grams daily) for 3 weeks. The same 3 exercise tests were repeated after 3 weeks.

\section{Results}

There was a significant increase in $\mathrm{VO}_{2} \max (44.0 \pm 10.5$ to $48.8 \pm 11.1 \mathrm{ml} / \mathrm{kg} / \mathrm{min}^{-1} ; \mathrm{p}=0.042$ ) for MR. There was also an increase in TTE $(+69.8 \mathrm{sec} ; 851.7 \pm 170.0$ to $921.5 \pm$ $146.2 \mathrm{sec}$ ) for MR as determined by $95 \%$ confidence intervals. No changes in $\mathrm{VO}_{2}$ max or TTE were observed for PL ( $p>0.05)$. Though not statistically significant $(\mathrm{p}>0.05)$, there was a greater increase in VT for MR $(+0.9 \mathrm{~L} / \mathrm{min} ; 1.7$ \pm 0.3 to $2.4 \pm 1.0 \mathrm{~L} / \mathrm{min})$ compared to $\mathrm{PL}(+0.2 \mathrm{~L} / \mathrm{min} ; 2.3$ \pm 0.9 to $2.5 \pm 0.7 \mathrm{~L} / \mathrm{min})$. Lactate increased significantly over the 6 min test in both groups with no significant difference between groups $(p=0.369)$. There was also a nonsignificant increase $(\mathrm{p}>0.05)$ in PP in MR $(51 \pm 113 \mathrm{~W})$ and a decrease in PP in PL $(-48 \pm 50 \mathrm{~W})$.

\section{Conclusion}

Chronic, 3 week supplementation of a mushroom blend at 4 grams per day may improve $\mathrm{VO}_{2}$ max increase TTE and augment PP during high-intensity aerobic exercise. A blend containing Cordyceps militaris may be an effective method for enhancing aerobic performance and delaying fatigue by improving oxygen kinetics. This could have positive implications for maintaining and improving training volume, especially in endurance athletes.

\section{Acknowledgements}

This study was funded by Scivation Inc.

Published: 21 September 2015

doi:10.1186/1550-2783-12-S1-P45

Cite this article as: Hirsch et al:: Chronic supplementation of a mushroom blend on oxygen kinetics, peak power, and time to exhaustion. Journal of the International Society of Sports Nutrition 201512 (Suppl 1):P45 